\title{
Molecular Imaging Reveals Trastuzumab-Induced Epidermal Growth Factor Receptor Downregulation In Vivo
}

\author{
Teng $\mathrm{Ma}^{1,2}$, Xianlei Sun ${ }^{1,2}$, Liyang Cui ${ }^{1,2}$, Liquan $\mathrm{Gao}^{1,2}$, Yue $\mathrm{Wu}^{1,2}$, Hao Liu ${ }^{1,2}$, Zhaohui Zhu ${ }^{3}$, Fan Wang ${ }^{1,2}$, \\ and Zhaofei Liu ${ }^{1,2}$ \\ ${ }^{I}$ Medical Isotopes Research Center, Peking University, Beijing, China; ${ }^{2}$ Department of Radiation Medicine, School of Basic Medical \\ Sciences, Peking University, Beijing, China; and ${ }^{3}$ Department of Nuclear Medicine, Peking Union Medical College Hospital, Beijing, \\ China
}

Previous in vitro studies demonstrated that treating tumors expressing both epidermal growth factor receptor (EGFR) and human epidermal growth factor receptor 2 with trastuzumab resulted in increased EGFR homodimerization and subsequent rapid downregulation of EGFR. We investigated whether molecular imaging using near-infrared fluorescence (NIRF) imaging and PET probes could sensitively detect trastuzumab-induced EGFR downregulation in vivo. Methods: The $F\left(a b^{\prime}\right)_{2}$ antibody fragment PaniF(ab') was generated by digesting the anti-EGFR monoclonal antibody panitumumab. PaniF $\left(a b^{\prime}\right)_{2}$ was labeled with either a NIRF dye or ${ }^{68} \mathrm{Ga}$, and optical imaging and small-animal PET imaging of DyePaniF $\left(a b^{\prime}\right)_{2}$ and ${ }^{68} \mathrm{Ga}-\operatorname{PaniF}\left(\mathrm{ab}^{\prime}\right)_{2}$, respectively, were performed in HT-29 tumor-bearing nude mice treated with trastuzumab or untreated control. Results: Longitudinal NIRF imaging studies revealed significantly reduced tumor uptake of Dye-PaniF $\left(a b^{\prime}\right)_{2}$ on days 5 and 7 in trastuzumab-treated HT-29 tumors, compared with control. Western blotting confirmed the downregulation of EGFR after treatment with trastuzumab. Small-animal PET on day 5 after trastuzumab treatment also demonstrated decreased ${ }^{68} \mathrm{Ga}-$ PaniF $\left(a b^{\prime}\right)_{2}$ uptake in trastuzumab-treated HT-29 tumors. The tumor uptake value of ${ }^{68} \mathrm{Ga}-\mathrm{PaniF}\left(\mathrm{ab}^{\prime}\right)_{2}$ obtained from PET imaging had an excellent linear correlation with the uptake value measured using biodistribution. Conclusion: The downregulation of EGFR induced by trastuzumab treatment could be detected noninvasively using optical and PET imaging. This molecular imaging strategy could provide a dynamic readout of changes in the tumor signaling and may facilitate the noninvasive monitoring of the early tumor response to drug treatment.

Key Words: molecular imaging; EGFR; HER2; immunotherapy; tumor response

J Nucl Med 2014; 55:1002-1007

DOI: 10.2967/jnumed.114.137000

$\mathbf{E}$ pidermal growth factor (EGF) receptor (EGFR, also known as ErbB1) and human epidermal growth factor receptor 2 (HER2; ErbB2) are members of the EGFR family of transmembrane protein tyrosine kinase receptors that also includes HER3 (ErbB3)

Received Jan. 2, 2014; revision accepted Mar. 11, 2014.

For correspondence contact: Zhaofei Liu, Medical Isotopes Research

Center, Peking University, 38 Xueyuan Rd., Beijing 100191, China.

E-mail: liuzf@bjmu.edu.cn.

Published online Apr. 14, 2014.

COPYRIGHT (C 2014 by the Society of Nuclear Medicine and Molecular Imaging, Inc. and HER4 (ErbB4) (1). EGFR is upregulated in a variety of human tumors, which is correlated with tumor proliferation and invasion (2). EGFR can be activated by specific ligands including EGF and transforming growth factor- $\alpha$. On activation by ligands, EGFR activates downstream signaling pathways by either forming homodimers or forming heterodimers with other EGFR family members $(1,2)$. Unlike EGFR, HER2 has no natural ligand, and the activation of HER2 is induced usually by heterodimerization with other EGFR family members. HER2 is overexpressed in up to $30 \%$ of breast cancers (1); its expression is usually associated with a more aggressive tumor phenotype, particularly when the tumor cells coexpress EGFR (3).

Trastuzumab (Herceptin; Genentech, Inc.) is a recombinant humanized monoclonal antibody against the extracellular domain of HER2 (4). Clinical studies using trastuzumab as an adjuvant therapy or in combination with chemotherapy have shown promising results (5). However, the therapeutic efficacy of trastuzumab has not met expectations so far. For example, less than $30 \%$ of patients with metastatic breast cancer respond to first-line trastuzumab therapy (6). Moreover, most patients who are initial responders eventually acquire resistance to trastuzumab therapy (7).

High levels of HER2 expression may be considered to be a pretreatment predictor of trastuzumab efficacy (8). In clinical practice, the HER2 status of tumor tissues can be assessed using immunohistochemistry for HER2 protein expression and fluorescence in situ hybridization for DNA amplification. However, both of these methods require tumor tissues that are obtained from biopsies, which is an invasive procedure that cannot be repeated frequently. Moreover, the results of immunohistochemistry and fluorescence in situ hybridization may be variable because of tumor heterogeneity (9). Another method to assess HER2 levels in cancer patients is to quantify serum HER2 expression using immunoassays (10). However, immunoassays do not provide whole-body information or report the HER2 levels in primary and metastatic lesions. In fact, it is not certain that all HER2positive $($ HER $2+$ ) tumors will benefit from trastuzumab therapy. Therefore, further studies are needed to develop novel approaches that could more accurately predict the efficacy of trastuzumab before commencing therapy or more sensitively identify responsive and nonresponsive tumors during trastuzumab therapy.

Molecular imaging is a robust tool for the noninvasive detection of biologic processes and monitoring the pharmacodynamics of biomarkers in vivo. HER2 was proposed as a specific biomarker for assessing the response of tumors to trastuzumab therapy. Although trastuzumab labeled with ${ }^{111} \mathrm{In}(11),{ }^{89} \mathrm{Zr}(12)$, and ${ }^{64} \mathrm{Cu}$ (13) has already been tested in humans for specific HER2 targeting, 
these radiotracers may not be optimal for monitoring the efficacy of trastuzumab treatment because of potential self-blocking and competitive inhibition after several rounds of trastuzumab therapy. To overcome this, McLarty et al. (14) developed ${ }^{111}$ In-labeled pertuzumab, an antibody that recognizes the distinct epitope of HER2 that is targeted by trastuzumab. This study demonstrated that the radiotracer could sensitively monitor trastuzumab-induced HER2 downregulation in vivo. In addition to pertuzumab, several other HER2-targeting agents that recognize distinct epitopes from trastuzumab, such as an ${ }^{124}$ I-labeled diabody (15) and an ${ }^{18} \mathrm{~F}$ labeled Affibody (16), were also developed. All of these radiotracers have reported high HER2 specificity, and imaging results demonstrated that the molecular imaging of HER2 expression is a sensitive biomarker for assessing the early tumor response to trastuzumab treatment.

EGFR is commonly expressed in HER2+ tumors, and the synergistic interaction between these 2 EGFR family members could lead to a more malignant phenotype and poor prognosis $(3,17,18)$. Using an engineered $\beta$-gal system, Wehrman et al. (19) reported that treating EGFR- and HER2-coexpressing cells in vitro with trastuzumab inhibited the formation of HER2-EGFR heterodimers and subsequently increased EGFR homodimer formation in the presence of EGF. The increased number of EGFR homodimers resulted in increased EGFR internalization and downregulation. On the basis of these findings, we hypothesized that molecular imaging of EGFR expression in vivo could be used for characterizing the process of trastuzumab-induced EGFR downregulation noninvasively and thereby monitoring tumor responses to trastuzumab. We used the $\mathrm{F}\left(\mathrm{ab}^{\prime}\right)_{2}$ fragment of panitumumab (Vectibix; Amgen, Inc.), a humanized anti-EGFR monoclonal antibody, as the EGFR-targeting agent, which was then labeled with either an optical imaging dye or ${ }^{68} \mathrm{Ga}$. The downregulation of EGFR induced by trastuzumab treatment in a preclinical animal model was assessed using optical and PET imaging.

\section{MATERIALS AND METHODS}

\section{Cell Culture and Animal Model}

HT-29 human colon cancer, A549 human lung carcinoma, and A431 human epidermoid carcinoma cell lines were obtained from American Type Culture Collection. Cells were grown in Dulbecco modified Eagle medium supplemented with $10 \%$ fetal bovine serum at $37^{\circ} \mathrm{C}$ in a humidified atmosphere containing $5 \% \mathrm{CO}_{2}$. All animal experiments were performed in accordance with the Institutional Animal Care and Use Committee at Peking University. To establish the HT-29 tumor model, $5 \times 10^{6}$ tumor cells were inoculated subcutaneously into the right front flanks or right thighs of female BALB/c nude mice. Tumor growth was measured using calipers, and tumor volume was calculated using the formula volume $=$ length $\times$ width $^{2} / 2$.

\section{Expression of EGFR and HER2 in Cell Lines}

Panitumumab and trastuzumab were radiolabeled with $\mathrm{Na}^{125} \mathrm{I}$ (Perkin-Elmer) as described previously (20). ${ }^{125} \mathrm{I}$-panitumumab and ${ }^{125} \mathrm{I}-$ trastuzumab were then used as the EGFR- and HER2-specific radioligands, respectively. The expression levels of EGFR and HER2 in HT-29, A549, and A431 cells were determined by radioligand saturation binding assays, as described previously (20). The results were expressed as the number of receptors per tumor cell.

\section{Effects of Trastuzumab on EGFR Expression In Vitro}

Serum-starved HT-29, A549, and A431 tumor cells were treated with or without trastuzumab $(25 \mu \mathrm{g} / \mathrm{mL})$ at $37^{\circ} \mathrm{C}$ for $2 \mathrm{~h}$, and human EGF (hEGF) $(20 \mathrm{ng} / \mathrm{mL})$ was added for the last $10 \mathrm{~min}$ before cells were harvested. After being washed with ice-cold phosphate-buffered saline (PBS), cells were lysed and analyzed by Western blotting to determine the EGFR levels.

\section{In Vivo Trastuzumab Therapy}

HT-29 tumor-bearing nude mice were separated into 2 groups and injected intraperitoneally with trastuzumab (200 $\mu \mathrm{g}$ per mouse) or PBS ( $n=9$ per group) for $5 \mathrm{~d}$ (from days 1 to 5 ). Tumor size and body weight were measured every other day. On day 5, 4 animals from each group were sacrificed, and tumor samples were harvested. Half of each tumor was frozen and then cut into 5 - $\mu \mathrm{m}$-thick sections for immunofluorescence staining. The remaining half of each tumor was lysed, and the EGFR levels were determined by Western blotting.

\section{Western Blotting}

Detailed procedures are described in the supplemental materials and methods (supplemental materials are available at http://jnm. snmjournals.org). After Western blotting, the images were opened in ImageJ (http://rsb.info.nih.gov/ij/). A region of interest (ROI) was drawn on the first lane, and the same size and shape ROI was applied to all other lanes and the background to quantify the intensity of each band.

\section{Longitudinal Near-Infrared Fluorescence (NIRF) Imaging of EGFR Expression}

The EGFR-targeting NIRF agent was generated by conjugating the $\mathrm{F}\left(\mathrm{ab}^{\prime}\right)_{2}$ fragment of panitumumab, named $\operatorname{PaniF}\left(\mathrm{ab}^{\prime}\right)_{2}$, with DyLight680-NHS ester (Pierce Biotechnology, Inc.). PaniF $\left(\mathrm{ab}^{\prime}\right)_{2}$ was generated by digesting panitumumab using a $\mathrm{F}\left(\mathrm{ab}^{\prime}\right)_{2}$ preparation kit (Pierce Biotechnology, Inc.). The identification of $\operatorname{PaniF}\left(\mathrm{ab}^{\prime}\right)_{2}$ was confirmed by sodium dodecyl sulfate polyacrylamide gel electrophoresis (Supplemental Fig. 1). The immunoreactive fraction of ${ }^{125} \mathrm{I}-$ labeled PaniF $\left(\mathrm{ab}^{\prime}\right)_{2}$ was measured to be $77.57 \% \pm 3.73 \%$ using HT29 cells by the method previously described (21). A cell competition binding assay also confirmed that the EGFR immunoreactivity of $\operatorname{PaniF}\left(\mathrm{ab}^{\prime}\right)_{2}$ was similar to that of intact panitumumab (Supplemental Fig. 2). PaniF $\left(a b^{\prime}\right)_{2}$ was conjugated with DyLight680-NHS using a previously described method (22). Detailed procedures are described in the supplemental materials and methods. The DyLight680-NHS$\operatorname{PaniF}\left(\mathrm{ab}^{\prime}\right)_{2}$ conjugate, named Dye-PaniF $\left(\mathrm{ab}^{\prime}\right)_{2}$, was stored at $4{ }^{\circ} \mathrm{C}$ for further in vivo applications.

Serial NIRF imaging of the tumor response to trastuzumab therapy was performed on days $0,3,5$, and 7 in the HT-29 tumor model. Mice were injected intravenously with $0.5 \mathrm{nmol}$ of Dye-PaniF $\left(\mathrm{ab}^{\prime}\right)_{2}$, and in vivo NIRF imaging was then performed $4 \mathrm{~h}$ after injection. After a baseline scan was obtained on day 0,5 daily treatments of trastuzumab (200 $\mu \mathrm{g}$ per mouse intraperitoneally) or PBS were administered on days 1,2 , 3,4 , and 5 in the treatment and control groups, respectively $(n=5$ per group). NIRF imaging was repeated on days 3, 5, and 7 under the same conditions. For each scan, mice were anesthetized by the inhalation of $2 \%$ isoflurane in oxygen; NIRF imaging was then performed using an IVIS small-animal imaging system (Xenogen) (excitation, $675 \mathrm{~nm}$, emission, $720 \mathrm{~nm}$ ) as previously described $(22,23)$. Identical illumination settings were used to acquire all scans. To quantify the tumor uptake of Dye-PaniF $\left(\mathrm{ab}^{\prime}\right)_{2}$, the ROI was drawn for each tumor using Living Image software (Xenogen). The fluorescence intensity was presented as the average radiant efficiency in the unit of $\left[\mathrm{p} / \mathrm{s} / \mathrm{cm}^{2} / \mathrm{sr}\right] /\left[\mu \mathrm{W} / \mathrm{cm}^{2}\right]$. Tumor uptake was then calculated by normalizing the fluorescence intensity of the tumor with the total injection dose.

\section{${ }^{68} \mathrm{Ga}$ Radiolabeling and Small-Animal PET Imaging}

$\mathrm{PaniF}\left(\mathrm{ab}^{\prime}\right)_{2}$ was conjugated to 1,4,7-triazacyclononane-1,4,7-triacetic acid (NOTA) under standard isothiocyanate-amine reaction conditions. NOTA-PaniF $\left(\mathrm{ab}^{\prime}\right)_{2}$ was then radiolabeled with ${ }^{68} \mathrm{Ga}$. Detailed procedures are described in the supplemental materials and methods. 


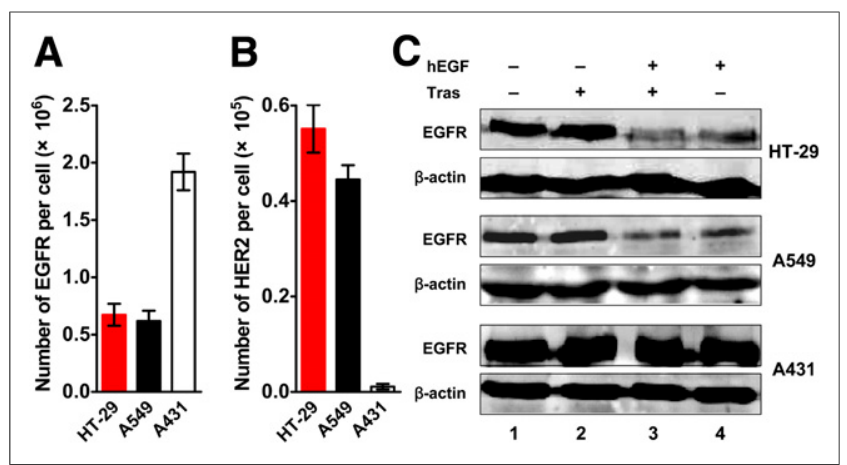

FIGURE 1. (A and B) Calculated amount of EGFR (A) and HER2 (B) per cell in 3 cell lines. (C) Western blotting of EGFR expression in tumor cells treated with or without trastuzumab, followed by presence or absence of hEGF. Tras $=$ trastuzumab.

HT-29 tumor-bearing nude mice were treated with trastuzumab (200 $\mu \mathrm{g}$ per mouse daily) or PBS by intraperitoneal injection for 5 $\mathrm{d}$ (from days 1 to 5). On day 5, mice were injected via the tail vein with $3.7 \mathrm{MBq}$ of ${ }^{68} \mathrm{Ga}-\mathrm{PaniF}\left(\mathrm{ab}^{\prime}\right)_{2}$ under isoflurane anesthesia. At $3 \mathrm{~h}$ after injection, 5-min static small-animal PET images were acquired ( $n=4$ per group). The ROI-derived percentage injected dose per gram of tissue $(\% \mathrm{ID} / \mathrm{g})$ was determined using a previously described method (24). For a blocking study, a group of 4 HT-29 tumor-bearing mice were injected intravenously with $1 \mathrm{mg}$ of panitumumab $4 \mathrm{~h}$ before injection of $3.7 \mathrm{MBq}$ of ${ }^{68} \mathrm{Ga}-\mathrm{PaniF}\left(\mathrm{ab}^{\prime}\right)_{2}$. At $3 \mathrm{~h}$ after injection with ${ }^{68} \mathrm{Ga}-\mathrm{PaniF}\left(\mathrm{ab}^{\prime}\right)_{2}$, the mice underwent PET scanning.

\section{Biodistribution of ${ }^{68} \mathrm{Ga}-\operatorname{PaniF}\left(\mathrm{ab}^{\prime}\right)_{2}$}

Immediately after PET imaging, HT-29 tumor-bearing nude mice were sacrificed and dissected. Blood, tumors, major organs, and tissues were harvested and weighed; the radioactivity in the tissues was then measured using a $\gamma$ counter (Packard). The results are presented as $\% \mathrm{ID} / \mathrm{g}$. The tumor and muscle uptake values of ${ }^{68} \mathrm{Ga}-\mathrm{PaniF}\left(\mathrm{ab}^{\prime}\right)_{2}$ determined by biodistribution were correlated with those determined by PET quantification using GraphPad Prism 4.0 (GraphPad Software). The correlation coefficient $\left(R^{2}\right)$ was then calculated applying a linear fit to measure the strength of the association.

\section{Statistical Analysis}

Quantitative data were expressed as mean \pm SD. Means were compared using the Student $t$ test. $P$ values of less than 0.05 were considered to statistically significant.

\section{RESULTS}

Trastuzumab-Induced EGFR Downregulation in EGFR- and HER2-Coexpressing Cell Lines

The radioligand binding assay demonstrated that HT-29 and A549 cells were EGFR-positive (EGFR+) and HER2+, whereas A431 cells expressed EGFR but were HER2-negative (HER2-) (Figs. 1A and 1B). HT-29 (EGFR+, HER2+), A549 (EGFR+, HER2+), and A431 (EGFR+, HER2-) cells were then used to investigate the effects of trastuzumab on the downregulation of EGFR. As shown in Figure 1C, EGFR expression was unaffected by trastuzumab treatment without hEGF stimulation in all 3 cell lines (lanes 1 and 2). However, in the presence of hEGF, trastuzumab treatment caused a $30 \%-50 \%$ reduction in EGFR expression in the EGFR + and HER2 + HT-29 and A549 cells (Fig. 1C, lane 3 vs. lane 4). Because A431 cells are HER2-, trastuzumab treatment did not result in the downregulation of EGFR expression, even in the presence of hEGF (Fig. 1C, lane 3 vs. lane 4).

\section{In Vivo Therapeutic Efficacy of Trastuzumab}

As shown in Figure 2A, trastuzumab treatment suppressed HT29 tumor growth. A difference in the average fractional tumor volumes $\left(\mathrm{V} / \mathrm{V}_{0}\right)$ between the trastuzumab and control groups was observed beginning on day 4 . On day 10 , trastuzumab treatment resulted in a $\mathrm{V} / \mathrm{V}_{0}$ of $2.43 \pm 0.85$, which was decreased significantly, compared with PBS control $\left(\mathrm{V} / \mathrm{V}_{0}=4.44 \pm 0.67\right.$, $P<0.05)$. Trastuzumab treatment did not cause any apparent body weight loss in the mice (Fig. 2B).

\section{Longitudinal NIRF Imaging of EGFR Expression Using Dye-PaniF $\left(\mathrm{ab}^{\prime}\right)_{2}$}

The in vivo EGFR-targeting efficacy of Dye-PaniF $\left(\mathrm{ab}^{\prime}\right)_{2}$ was confirmed in a blocking study in HT-29 tumor-bearing nude mice (Supplemental Fig. 3). Serial NIRF imaging studies (on days 0, 3,

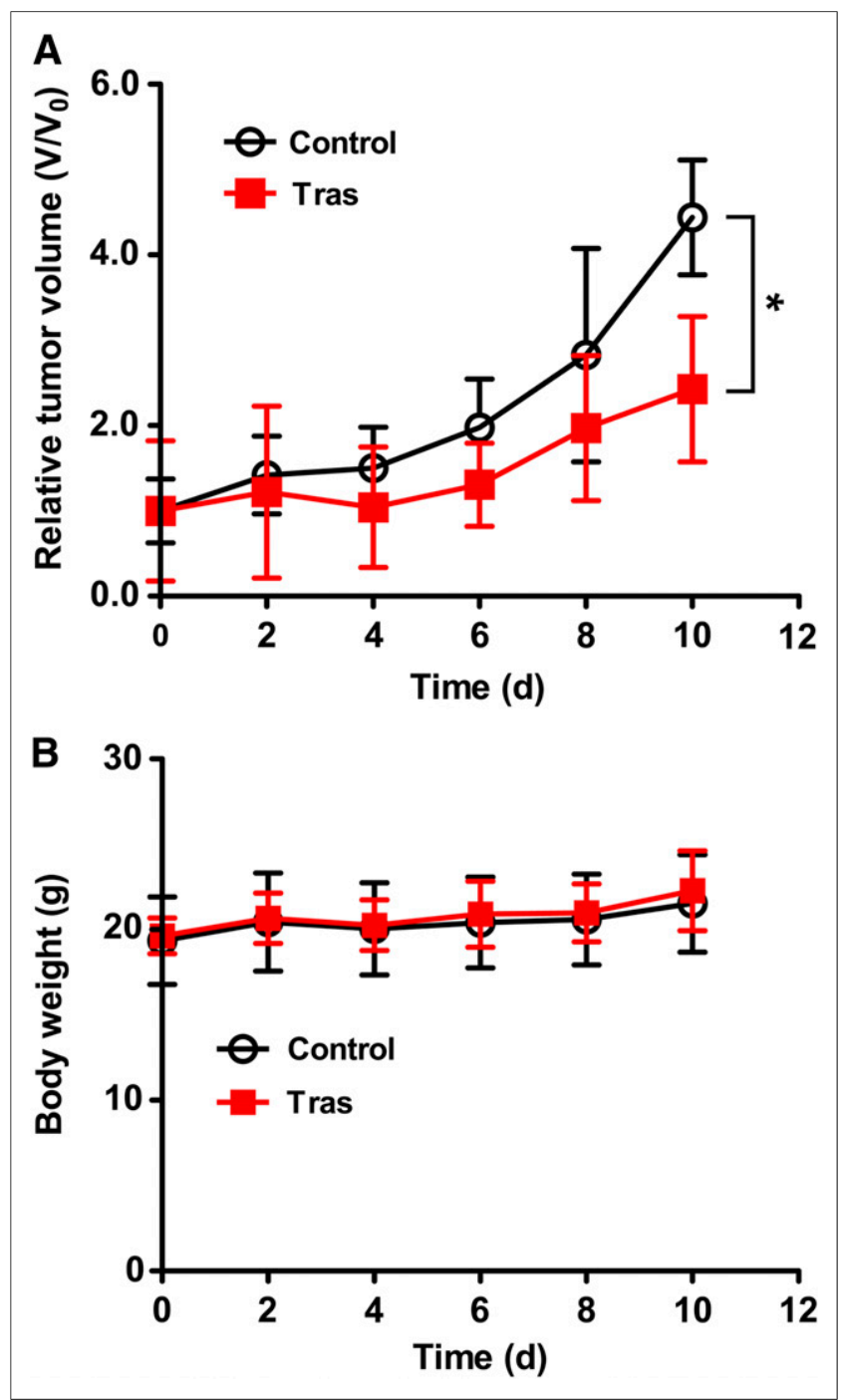

FIGURE 2. Tumor growth curve $(\mathrm{A})$ and mean body weight over time (B) of HT-29 tumor-bearing nude mice treated daily for $5 \mathrm{~d}$ with trastuzumab (Tras) or PBS (control). ${ }^{*} P<0.05$. 


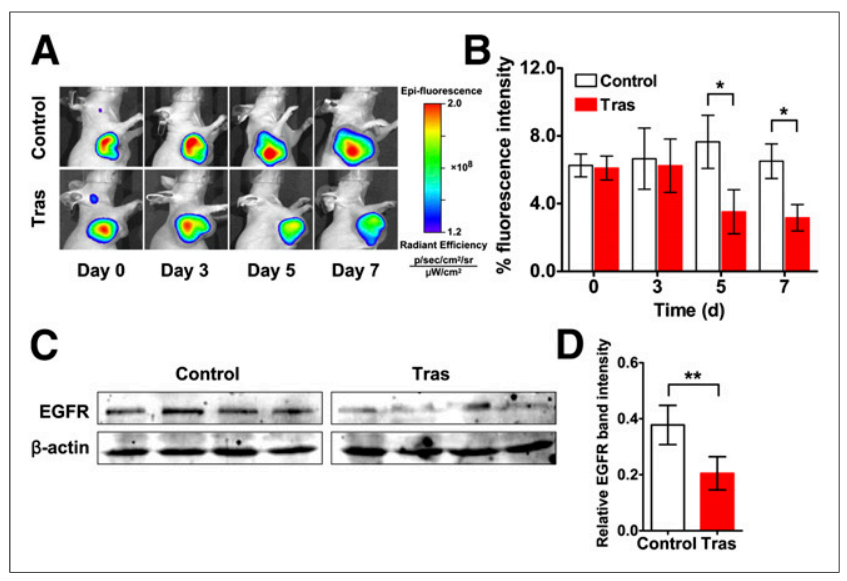

FIGURE 3. ( $A$ and $B$ ) Longitudinal in vivo optical images ( $A$ ) and quantified tumor uptake (B) of Dye-PaniF $\left(\mathrm{ab}^{\prime}\right)_{2}$ in $\mathrm{HT}-29$ tumor-bearing nude mice at $4 \mathrm{~h}$ after injection on days $0,3,5$, and 7 after treatment with trastuzumab (Tras) or PBS (control) daily for $5 \mathrm{~d}$. (C) Western blotting of EGFR expression in HT-29 tumor tissues of nude mice on day 5 after treatment with trastuzumab or PBS (control) daily for $5 \mathrm{~d}$. (D) Relative EGFR band intensity quantified from $C .{ }^{*} P<0.05$. ${ }^{\star \star} P<0.01$.

5, and 7) using Dye-PaniF $\left(\mathrm{ab}^{\prime}\right)_{2}$ were then performed to determine trastuzumab treatment-induced changes in EGFR expression in the HT-29 tumor mouse model. As shown in Figure 3A, reduced tumor fluorescence signals were observed on days 5 and 7 in the trastuzumab treatment group, compared with control. The quantified percentage tumor fluorescence intensity of Dye-PaniF $\left(\mathrm{ab}^{\prime}\right)_{2}$ in the trastuzumab-treated tumors was significantly lower than that in control tumors on days $5(3.52 \% \pm 1.30 \%$ vs. $7.65 \% \pm 1.57 \%$, $P<0.05)$ and $7(3.17 \% \pm 0.78 \%$ vs. $6.51 \% \pm 1.02 \%, P<0.05)$ (Fig. 3B).

To confirm the EGFR downregulation identified using NIRF imaging, ex vivo fluorescence staining and Western blotting studies were performed. HT-29 tumor tissues were harvested from nude mice after $5 \mathrm{~d}$ of trastuzumab treatment, frozen cut, and stained using EGFR-specific fluorescein isothiocyanate-panitumumab (a detailed procedure is provided in the supplemental materials and methods). Compared with the control group, fluorescein isothiocyanate-panitumumab staining was reduced significantly in trastuzumab-treated HT-29 tumors (Supplemental Fig. 4). Western blotting was then performed to further quantify the EGFR levels in the HT-29 tumors. As shown in Figures 3C and 3D, the relative EGFR band intensity in the trastuzumab-treated HT-29 tumors was significantly lower than in the control tumors $(0.21 \pm 0.06$ vs. $0.38 \pm 0.07, P<0.01)$. Taken together, these data confirm the downregulation of EGFR after trastuzumab treatment.

\section{Small-Animal PET and Biodistribution of ${ }^{68} \mathrm{Ga}-\mathrm{PaniF}\left(\mathrm{ab}^{\prime}\right)_{2}$}

Trastuzumab-induced EGFR downregulation was investigated further by small-animal PET and biodistribution using ${ }^{68} \mathrm{Ga}-\mathrm{PaniF}$ $\left(\mathrm{ab}^{\prime}\right)_{2}$. On day $5,{ }^{68} \mathrm{Ga}-\mathrm{PaniF}\left(\mathrm{ab}^{\prime}\right)_{2}$ PET imaging and biodistribution analysis were performed. As shown in Figure 4A, ${ }^{68} \mathrm{Ga}-\mathrm{PaniF}$ $\left(\mathrm{ab}^{\prime}\right)_{2}$ was visualized clearly in the tumors of the control group. In contrast, decreased ${ }^{68} \mathrm{Ga}-\mathrm{PaniF}\left(\mathrm{ab}^{\prime}\right)_{2}$ uptake was observed in trastuzumab-treated tumors. Importantly, no apparent tumor accumulation of ${ }^{68} \mathrm{Ga}-\mathrm{PaniF}\left(\mathrm{ab}^{\prime}\right)_{2}$ was observed in the tumors blocked by competitive inhibition. The uptake values in tumors were measured from ROI analyses and are shown in Figure 4B. The uptake of ${ }^{68} \mathrm{Ga}-\mathrm{PaniF}\left(\mathrm{ab}^{\prime}\right)_{2}$ in trastuzumab-treated tumors was signifi- cantly lower than in the control tumors $(1.15 \pm 0.42$ vs. $2.70 \pm$ $0.51 \% \mathrm{ID} / \mathrm{g}, P<0.01)$. After blocking with cold panitumumab, the tumor uptake of ${ }^{68} \mathrm{Ga}-\mathrm{PaniF}\left(\mathrm{ab}^{\prime}\right)_{2}$ was inhibited significantly (from $2.70 \pm 0.51$ to $0.77 \pm 0.15 \% \mathrm{ID} / \mathrm{g}, P<0.001$ ).

To validate the accuracy of the PET quantification, a necropsybased biodistribution experiment was performed. As shown in Figure $4 \mathrm{C}$, predominant uptake of ${ }^{68} \mathrm{Ga}-\mathrm{PaniF}\left(\mathrm{ab}^{\prime}\right)_{2}$ was observed in the blood and liver in both control and trastuzumab groups. However, the uptake of ${ }^{68} \mathrm{Ga}-\mathrm{PaniF}\left(\mathrm{ab}^{\prime}\right)_{2}$ in trastuzumab-treated HT-29 tumors was significantly lower than in control tumors $(1.58 \pm 0.16$ vs. $2.44 \pm 0.30 \% \mathrm{ID} / \mathrm{g}, P<0.01)$. The values obtained from quantified PET images were then compared with the values derived from the biodistribution study. As shown in Figure 4D, a linear relationship was found between the ${ }^{68} \mathrm{Ga}-\mathrm{PaniF}\left(\mathrm{ab}^{\prime}\right)_{2}$ uptake quantified using PET ROI analysis and the necropsy-based biodistribution study, with a correlation coefficient $\left(R^{2}\right)$ of 0.80 . These data demonstrated the accuracy of noninvasive PET imaging to quantify the downregulation of EGFR after trastuzumab treatment.

\section{DISCUSSION}

Although molecular imaging techniques have been extensively investigated in the field of oncology, relatively few approaches were applied to provide a deeper understanding of the biologic dynamics of tumor progression that extends beyond merely mapping the density of a target and localizing the lesions (25). Recently, pioneering works have been conducted to measure the androgen receptor pathway $(26,27)$ and the aberrant MYC signaling (28) using PET. These studies demonstrated that molecular imaging of a biomarker that is overexpressed in tumors enables the systematic measurement of oncogenic signaling pathways associated with therapeutic interventions $(25,29)$.

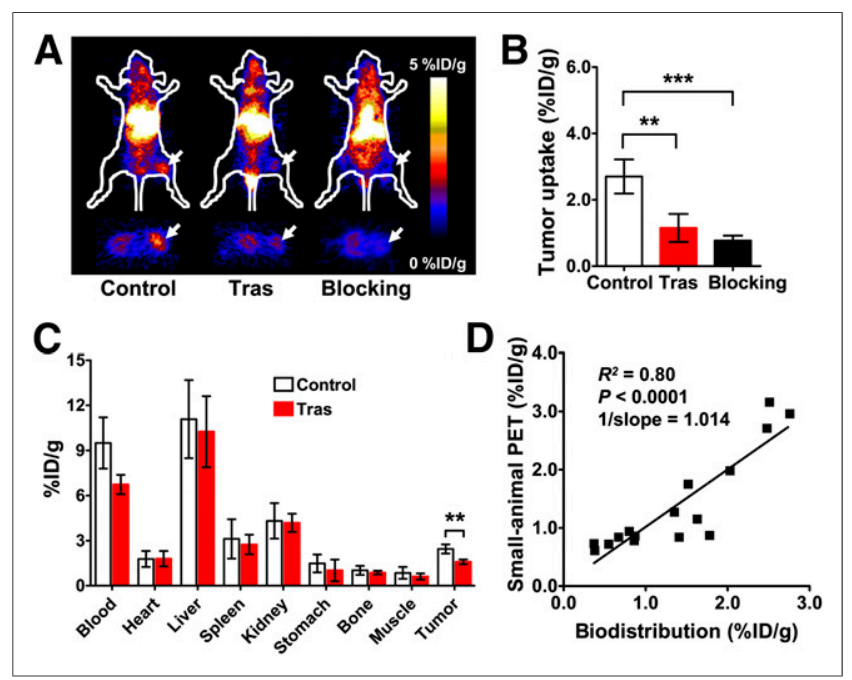

FIGURE 4. (A and B) Decay-corrected whole-body coronal (top) and transaxial (bottom) PET images (A) and quantified tumor uptake (B) of ${ }^{68} \mathrm{Ga}-\mathrm{PaniF}\left(\mathrm{ab}^{\prime}\right)_{2}$ in $\mathrm{HT}-29$ tumor-bearing nude mice at $3 \mathrm{~h}$ after injection on day 5 after treatment with trastuzumab (Tras) or PBS (control; with or without panitumumab blocking) daily for $5 \mathrm{~d}$. (C) Necropsy-based biodistribution of ${ }^{68} \mathrm{Ga}-\mathrm{PaniF}\left(\mathrm{ab} \mathrm{b}_{2}\right)_{2}$ in HT-29 tumor-bearing mice after PET scanning. (D) Correlation between PET-based quantification and biodistribution of ${ }^{68} \mathrm{Ga}-\mathrm{PaniF}\left(\mathrm{ab}^{\prime}\right)_{2} .{ }^{* \star} P<0.01$. ${ }^{* \star} P<0.001$. 


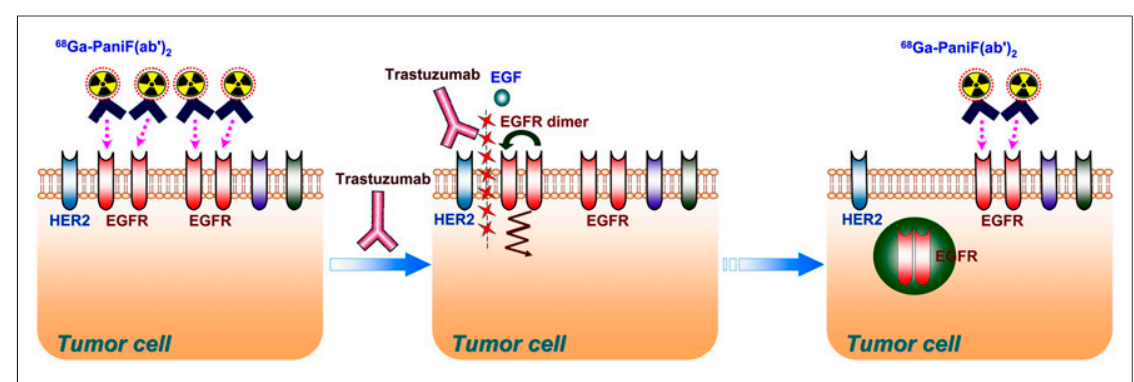

FIGURE 5. Schematic diagram of proposed mechanism for trastuzumab-induced EGFR downregulation and rationale of using EGFR-targeting radiotracer for noninvasive imaging of trastuzumab-induced EGFR downregulation.

In this study, we documented that the use of molecular imaging could quantitatively reflect trastuzumab treatment-induced changes in EGFR expression in vivo. Molecular imaging of the feedback loop interaction between EGFR and HER2, the 2 well-established tumor biomarkers of the EGFR family, could facilitate better understanding of the EGFR signaling pathway and cross-talks between EGFR family members. The dynamic readout of changes in tumor EGFR signaling on anti-HER2 treatment would also open up the possibility for early assessment of tumor response to therapy.

HER2 has been used as a biomarker for monitoring the treatment efficacy of trastuzumab in preclinical studies. Several PET radiotracers targeting HER2 have been investigated to determine the expression levels of HER2 noninvasively, thereby assessing the early tumor response to trastuzumab (14-16). However, data are controversial, because several studies questioned whether trastuzumab treatment downregulated HER2 (14,15,30,31). Possible explanations for these controversial results include the variability of different cell lines and tumor tissues and the insensitivity of immunohistochemistry staining methods for measuring HER2 levels (14). Because HER2 is the direct target of trastuzumab, the actions of trastuzumab on HER2 are likely to be dynamic, depending on the rapid internalization and reexpression of HER2.

A previous study demonstrated that trastuzumab could induce the downregulation of EGFR in cells coexpressing EGFR and HER2 (19). We confirmed this observation using 3 cell lines: HT29 (EGFR +, HER 2 +), A549 (EGFR+, HER2+), and A431 (EGFR+, HER2-). In HT-29 and A549 cells, EGFR levels were downregulated significantly after treatment with trastuzumab (in the presence of hEGF). In contrast, in A431 cells, which do not express HER2 but express high levels of EGFR, EGFR was not downregulated in response to trastuzumab treatment (Fig. 1). These results confirmed that trastuzumab induced the downregulation of EGFR in cells that coexpress EGFR and HER2. The hypothesized mechanism for these effects is that treating tumor cells expressing both EGFR and HER2 with trastuzumab could inhibit the formation of HER2-EGFR heterodimers; in the presence of EGF, the formation of EGFR homodimer would then be increased. The increased EGFR homodimer formation would result in increased internalization and downregulation of EGFR (Fig. 5).

Although EGFR is not the direct target of trastuzumab, it could indirectly reflect the binding of trastuzumab to HER2. Therefore, we sought to determine whether the molecular imaging of EGFR could indirectly reflect the action of trastuzumab on HER2. To achieve this, we first assessed whether the EGFR-targeting agent panitumumab cross-reacted with trastuzumab. If these agents cross-reacted, labeled panitumumab might not accurately detect
EGFR levels after repeated treatment with trastuzumab. The cell-binding assay confirmed that the 2 antibodies did not crossreact (Supplemental Fig. 5). We next performed longitudinal optical imaging to determine the dynamic changes in EGFR. During treatment with trastuzumab, the tumor uptake of EGFR-specific Dye$\operatorname{PaniF}\left(\mathrm{ab}^{\prime}\right)_{2}$ decreased by day 5, and the downregulation of EGFR was validated by fluorescence staining and Western blotting. Previously, Cai et al. (32) demonstrated that the EGFR levels could be determined noninvasively by PET using radiolabeled anti-EGFR antibody. The tumor uptake measured using PET imaging correlated with EGFR expression quantified using Western blotting. Compared with PET, optical imaging has limitations in tissue penetration and in image reconstruction and quantification. Therefore, we validated the optical imaging results with Dye-PaniF $\left(\mathrm{ab}^{\prime}\right)_{2}$ using PET imaging with the radiolabeled counterpart ${ }^{68} \mathrm{Ga}-\mathrm{PaniF}\left(\mathrm{ab}^{\prime}\right)_{2}$. The PET results confirmed the optical imaging data, in which trastuzumab treatment resulted in decreased tumor uptake of $\operatorname{PaniF}\left(\mathrm{ab}^{\prime}\right)_{2}$. Importantly, noninvasive imaging-based tumor quantification showed an excellent linear correlation with the biodistribution data, demonstrating that the molecular imaging analyses used in this study could detect EGFR expression levels in vivo noninvasively. The decreased EGFR expression was also confirmed in an A549 tumor mouse model (Supplemental Fig. 6).

Dynamic measurement of changes in EGFR expression using the methods described in this study may also facilitate the early assessment of tumor response to trastuzumab therapy. In vivo NIRF imaging manifested a significant reduction in the Dye-PaniF $\left(\mathrm{ab}^{\prime}\right)_{2}$ tumor uptake, compared with the control group, on day 5 after trastuzumab treatment (Fig. 3), which is 5 d earlier than the significant decrease observed in tumor size (day 10; Fig. 2A). Because EGFR-targeted therapies are a general strategy for tumor treatment, the technique developed in this study may also have applications for investigating the underlying mechanisms by which antitumor drugs modulate EGFR. Molecular imaging of EGFR to predict tumor responses to trastuzumab is limited to tumors that express both EGFR and HER2. In the clinical setting, the coexpression of EGFR and HER2 usually stands for a more malignant cancer phenotype $(3,17,18)$. Therefore, using molecular imaging of the pharmacodynamics of EGFR to predict the tumor response to trastuzumab might have possible clinical applications for aggressive tumor phenotypes.

\section{CONCLUSION}

Using optical and small-animal PET imaging, we were able to noninvasively assess the EGFR downregulation induced by trastuzumab treatment. This noninvasive molecular imaging strategy may facilitate the determination of trastuzumab-induced EGFR downregulation, investigation of the underlying mechanism, and detection of biologic behaviors in vivo.

\section{DISCLOSURE}

The costs of publication of this article were defrayed in part by the payment of page charges. Therefore, and solely to indicate this fact, this article is hereby marked "advertisement" in accordance with 18 USC section 1734. This work was supported, in part, by the 
National Natural Science Foundation of China (NSFC) projects (81222019, 81125011, 30930030, 81000625, and 81201127), "973" projects (2013CB733802 and 2011CB707703), grants from the Ministry of Science and Technology of China (2011YQ030114, 2012ZX09102301-018, and 2012BAK25B03-16), grants from the Ministry of Education of China (31300 and BMU20110263), grants from the Beijing Natural Science Foundation (7132131 and 7132123), and a grant from the Beijing Nova Program (Z121107002512010). No other potential conflict of interest relevant to this article was reported.

\section{REFERENCES}

1. Olayioye MA, Neve RM, Lane HA, Hynes NE. The ErbB signaling network: receptor heterodimerization in development and cancer. EMBO J. 2000;19:31593167.

2. Mendelsohn J, Baselga J. The EGF receptor family as targets for cancer therapy. Oncogene. 2000;19:6550-6565.

3. Slamon DJ, Clark GM, Wong SG, Levin WJ, Ullrich A, McGuire WL. Human breast cancer: correlation of relapse and survival with amplification of the HER2/neu oncogene. Science. 1987;235:177-182.

4. Hudis CA. Trastuzumab: mechanism of action and use in clinical practice. $N$ Engl J Med. 2007;357:39-51.

5. Slamon DJ, Leyland-Jones B, Shak S, et al. Use of chemotherapy plus a monoclonal antibody against HER2 for metastatic breast cancer that overexpresses HER2. N Engl J Med. 2001;344:783-792.

6. Vogel CL, Cobleigh MA, Tripathy D, et al. Efficacy and safety of trastuzumab as a single agent in first-line treatment of HER2-overexpressing metastatic breast cancer. J Clin Oncol. 2002;20:719-726.

7. Tagliabue E, Balsari A, Campiglio M, Pupa SM. HER2 as a target for breast cancer therapy. Expert Opin Biol Ther. 2010;10:711-724.

8. Ghosh R, Narasanna A, Wang SE, et al. Trastuzumab has preferential activity against breast cancers driven by HER2 homodimers. Cancer Res. 2011;71:1871-1882.

9. Marusyk A, Polyak K. Tumor heterogeneity: causes and consequences. Biochim Biophys Acta. 2010;1805:105-117.

10. Carney WP, Neumann R, Lipton A, Leitzel K, Ali S, Price CP. Potential clinical utility of serum HER-2/neu oncoprotein concentrations in patients with breast cancer. Clin Chem. 2003;49:1579-1598.

11. Perik PJ, Lub-De Hooge MN, Gietema JA, et al. Indium-111-labeled trastuzumab scintigraphy in patients with human epidermal growth factor receptor 2positive metastatic breast cancer. J Clin Oncol. 2006;24:2276-2282.

12. Dijkers EC, Oude Munnink TH, Kosterink JG, et al. Biodistribution of ${ }^{89} \mathrm{Zr}$ trastuzumab and PET imaging of HER2-positive lesions in patients with metastatic breast cancer. Clin Pharmacol Ther. 2010;87:586-592.

13. Tamura K, Kurihara H, Yonemori K, et al. ${ }^{64} \mathrm{Cu}$-DOTA-trastuzumab PET imaging in patients with HER2-positive breast cancer. J Nucl Med. 2013;54:1869-1875.

14. McLarty K, Cornelissen B, Cai Z, et al. Micro-SPECT/CT with ${ }^{111}$ In-DTPApertuzumab sensitively detects trastuzumab-mediated HER2 downregulation and tumor response in athymic mice bearing MDA-MB-361 human breast cancer xenografts. J Nucl Med. 2009;50:1340-1348.

15. Reddy S, Shaller CC, Doss M, et al. Evaluation of the anti-HER2 C6.5 diabody as a PET radiotracer to monitor HER2 status and predict response to trastuzumab treatment. Clin Cancer Res. 2011;17:1509-1520.
16. Kramer-Marek G, Gijsen M, Kiesewetter DO, et al. Potential of PET to predict the response to trastuzumab treatment in an ErbB2-positive human xenograft tumor model. J Nucl Med. 2012;53:629-637.

17. DiGiovanna MP, Stern DF, Edgerton SM, Whalen SG, Moore D 2nd, Thor AD. Relationship of epidermal growth factor receptor expression to ErbB-2 signaling activity and prognosis in breast cancer patients. J Clin Oncol. 2005;23:11521160 .

18. Lee-Hoeflich ST, Crocker L, Yao E, et al. A central role for HER3 in HER2amplified breast cancer: implications for targeted therapy. Cancer Res. 2008;68: 5878-5887.

19. Wehrman TS, Raab WJ, Casipit CL, Doyonnas R, Pomerantz JH, Blau HM. A system for quantifying dynamic protein interactions defines a role for Herceptin in modulating ErbB2 interactions. Proc Natl Acad Sci USA. 2006;103:1906319068.

20. Liu Z, Yu Z, He W, Ma S, Sun L, Wang F. In-vitro internalization and in-vivo tumor uptake of anti-EGFR monoclonal antibody LA22 in A549 lung cancer cells and animal model. Cancer Biother Radiopharm. 2009;24:15-24.

21. Lindmo T, Boven E, Cuttitta F, Fedorko J, Bunn PA Jr. Determination of the immunoreactive fraction of radiolabeled monoclonal antibodies by linear extrapolation to binding at infinite antigen excess. J Immunol Methods. 1984;72: 77-89.

22. Liu Z, Sun X, Liu H, et al. Early assessment of tumor response to gefitinib treatment by noninvasive optical imaging of tumor vascular endothelial growth factor expression in animal models. J Nucl Med. March 17, 2014 [Epub ahead of print].

23. Sun X, Ma T, Liu H, et al. Longitudinal monitoring of tumor antiangiogenic therapy with near-infrared fluorophore-labeled agents targeted to integrin alphabeta and vascular endothelial growth factor. Eur J Nucl Med Mol Imaging. February 22, 2014 [Epub ahead of print].

24. Liu Z, Li ZB, Cao Q, Liu S, Wang F, Chen X. Small-animal PET of tumors with ${ }^{64} \mathrm{Cu}$-labeled RGD-bombesin heterodimer. J Nucl Med. 2009;50:1168-1177.

25. Holland JP, Cumming P, Vasdev N. PET of signal transduction pathways in cancer. J Nucl Med. 2012;53:1333-1336.

26. Evans MJ, Smith-Jones PM, Wongvipat J, et al. Noninvasive measurement of androgen receptor signaling with a positron-emitting radiopharmaceutical that targets prostate-specific membrane antigen. Proc Natl Acad Sci USA. 2011;108: 9578-9582.

27. Ulmert D, Evans MJ, Holland JP, et al. Imaging androgen receptor signaling with a radiotracer targeting free prostate-specific antigen. Cancer Discov. 2012;2: 320-327.

28. Holland JP, Evans MJ, Rice SL, Wongvipat J, Sawyers CL, Lewis JS. Annotating MYC status with ${ }^{89} \mathrm{Zr}$-transferrin imaging. Nat Med. 2012;18:1586-1591.

29. Evans MJ. Measuring oncogenic signaling pathways in cancer with PET: an emerging paradigm from studies in castration-resistant prostate cancer. Cancer Discov. 2012;2:985-994.

30. Burstein HJ, Harris LN, Gelman R, et al. Preoperative therapy with trastuzumab and paclitaxel followed by sequential adjuvant doxorubicin/cyclophosphamide for HER2 overexpressing stage II or III breast cancer: a pilot study. J Clin Oncol. 2003;21:46-53.

31. Gennari R, Menard S, Fagnoni F, et al. Pilot study of the mechanism of action of preoperative trastuzumab in patients with primary operable breast tumors overexpressing HER2. Clin Cancer Res. 2004;10:5650-5655.

32. Cai W, Chen K, He L, Cao Q, Koong A, Chen X. Quantitative PET of EGFR expression in xenograft-bearing mice using ${ }^{64} \mathrm{Cu}$-labeled cetuximab, a chimeric anti-EGFR monoclonal antibody. Eur J Nucl Med Mol Imaging. 2007;34:850858. 